Pacific Journal of Mathematics

THE NUMBER OF EQUATIONS DEFINING POINTS IN 


\title{
THE NUMBER OF EQUATIONS DEFINING POINTS IN GENERAL POSITION
}

\author{
TIM SAUER
}

\begin{abstract}
Bounds are established for the number of generators of the graded homogeneous ideal of a set of points in generic or in uniform position in the projective plane. For $n \leq 11, n$ points in uniform position must have the "general" number of generators. It is shown by example that this fails for $n=12$.
\end{abstract}

Introduction. Let $Z$ be a set of points in $\mathbf{P}_{k}^{2}, k$ algebraically closed. We say the points of $Z$ lie in generic position if $Z$ imposes independent conditions on curves containing it. If this holds for all subsets of $Z$ we say that $Z$ lies in uniform position. Given a set $Z$ in one of these types of "general position", one would like to count the number of equations needed to cut out $Z$, or more precisely, the minimal number of generators $\nu$ of the graded homogeneous ideal $I(Z)$.

This question has arisen most recently in calculations of the CohenMacaulay-type of singularities. For example, it is shown in [7] that if $A$ is the local ring at a curve singularity $P$ in $\mathbf{A}_{k}^{3}$, and if the lines of the tangent cone at $P$ correspond to a set of distinct points $Z$ in generic position in $\mathbf{P}_{k}^{2}$, then the Cohen-Macaulay-type of $A$ is equal to $\nu(I(Z))-1$. It is then natural to look for geometric conditions on $Z$ which will allow the Cohen-Macaulay-type to be computed.

Let $s$ denote the number of points belonging to $Z, d$ the integer such that $\left(\begin{array}{c}d+1 \\ 2\end{array}\right) \leq s<\left(\begin{array}{c}d+2 \\ 2\end{array}\right)$, and define

$$
N(s)=\left\{\begin{array}{cl}
d+1-s+\left(\begin{array}{c}
d+1 \\
2
\end{array}\right) & \text { if }\left(\begin{array}{c}
d+1 \\
2
\end{array}\right) \leq s \leq \frac{d(d+2)}{2} \\
d+2+s-\left(\begin{array}{c}
d+2 \\
2
\end{array}\right) & \text { if } \frac{d(d+2)}{2} \leq s<\left(\begin{array}{c}
d+2 \\
2
\end{array}\right) .
\end{array}\right.
$$

Geramita and Maroscia [6] have shown that almost all sets of $s$ points in $\mathbf{P}^{2}$ are defined by exactly $N(s)$ equations. We give a new proof of this fact (1.7). However, $\nu(I(Z))$ is not constant on the sets of $s$ points in generic position. It follows from a theorem of Dubreil [4] that the best one can say is that if $Z$ lies in generic position, then $N(s) \leq \nu(I(Z)) \leq d+1$. 
Uniform position was introduced in [7] as a more stringent condition on $Z$; it is known that $\nu(I(Z))=N(s)$ if $Z$ is a set of $s$ points in uniform position and $s \leq 11$. If $s=12$, then $N(s)=3$, but we construct in $\S 2$ a set $Z$ of 12 points in uniform position for which $\nu(I(Z))=4$. Therefore, in a sense, some sets of points in uniform position are "more general" than others. We also establish an upper bound for $\nu(I(Z))$, where $Z$ lies in uniform position:

$$
\begin{array}{ll}
N(s) \leq \nu(I(Z)) \leq d \quad \text { if }\left(\begin{array}{c}
d+1 \\
2
\end{array}\right)<s<\left(\begin{array}{c}
d+2 \\
2
\end{array}\right)-1 \\
N(s)=\nu(I(Z))=d+1 \quad \text { if } s=\left(\begin{array}{c}
d+1 \\
2
\end{array}\right) \text { or }\left(\begin{array}{c}
d+2 \\
2
\end{array}\right)-1 .
\end{array}
$$

For example, if $s=12$, this result shows that $3 \leq \nu \leq 4$.

The author is grateful to W. C. Brown for many valuable conversations.

\section{Bounds on the number of generators.}

Definition. Let $Z$ be a set of $s$ points contained in $\mathbf{P}^{2}=\mathbf{P}_{k}^{2}, k$ an algebraically closed field, and $\mathscr{I}_{Z}$ its sheaf of ideals. We say $Z$ lies in generic position if for every nonnegative integer $m, \operatorname{dim} H^{0}\left(\mathscr{I}_{Z}(m)\right)=$ $\max \left\{0,\left(\begin{array}{c}m+2 \\ 2\end{array}\right)-s\right\}$, where $\mathscr{I}_{Z}(m)$ denotes $\mathscr{I}_{Z}$ twisted $m$ times by the hyperplane line bundle. We say $Z$ lies in uniform position if each subset of $Z$ (including $Z$ itself) lies in generic position.

REMARK 1.0.1. Roughly speaking, $Z$ lies in generic position if $Z$ imposes independent conditions on curves containing it. The sets of $s$ points in generic position in $\mathbf{P}^{2}$ form a Zariski-open subset of the Hilbert scheme Hilbs $\left(\mathbf{P}^{2}\right)$ parametrizing subschemes of $\mathbf{P}^{2}$ of length $s$. The sets of $s$ points in uniform position form an open subset of the sets of $s$ points in generic position.

Let $Z$ be a zero-dimensional subscheme of $\mathbf{P}^{2}$ of length $s$. Because the projective dimension of an ideal sheaf in $\mathbf{P}^{n}$ is at most $n-1, I_{Z}$ has a minimal projective resolution

$$
0 \rightarrow \sum_{i=1}^{\nu-1} \mathcal{O}_{\mathbf{P}^{2}}\left(-t_{i}\right) \stackrel{A}{\rightarrow} \sum_{j=1}^{\nu} \mathcal{O}_{\mathbf{P}^{2}}\left(-r_{j}\right) \stackrel{\left(f_{1}, \ldots, f_{\nu}\right)}{\rightarrow} \mathscr{I}_{Z} \rightarrow 0
$$

where $A$ is a $(\nu-1) \times \nu$ "relations matrix" of homogeneous forms of degrees $\alpha_{i j}=t_{i}-r_{j}$, and we arrange $t_{1} \leq \cdots \leq t_{\nu-1}$ and $r_{1} \leq \cdots \leq r_{\nu}$. A standard Chern class calculation shows $\sum_{i=1}^{\nu-1} t_{i}=\sum_{j=1}^{\nu} r_{j}$ and $2 s=$ $\sum_{i=1}^{\nu-1} t_{i}^{2}-\sum_{j=1}^{\nu} r_{j}^{2}$. Further, the minimality of the resolution implies that 
the entries of $A$ are in the irrelevant ideal, so we have $t_{1}>r_{1}$ and $t_{\nu-1}>r_{\nu}$.

LEMMA 1.1 (Burch, [3]). If I is an ideal of projective dimension one in a regular local ring $(R, \mathscr{M})$, then given a minimal resolution

$$
0 \rightarrow R^{n-1} \stackrel{A}{\rightarrow} R^{n} \stackrel{\left(f_{1}, \ldots, f_{n}\right)}{\rightarrow} I \rightarrow 0
$$

there exists $r \in R$ such that $f_{i}=r \Delta_{i}, i=1, \ldots, n$, where the $\Delta_{i}$ are the maximal minors of the matrix $A$.

The lemma applies in our case with $R=k\left[x_{0}, x_{1}, x_{2}\right]_{\left(x_{0}, x_{1}, x_{2}\right)}$ and $I(Z)=\sum_{m=0}^{\infty} H^{0}\left(\mathscr{I}_{Z}(m)\right)$ localized at $\left(x_{0}, x_{1}, x_{2}\right)$ for $I$. Since ht $I=2, r$ must be a unit, and we may assume in the following that $f_{i}=\Delta_{i}$.

EXAMPLE 1.1.1. If $Z$ is a complete intersection of curves of degrees $r_{1}$ and $r_{2}$, then the minimal resolution is the familiar Koszul complex

$$
0 \rightarrow \mathcal{O}_{\mathbf{P}^{2}}\left(-r_{1}-r_{2}\right) \rightarrow \mathcal{O}_{\mathbf{P}^{2}}\left(-r_{1}\right) \oplus \mathcal{O}_{\mathbf{P}^{2}}\left(-r_{2}\right) \stackrel{\left(f_{1}, f_{2}\right)}{\rightarrow} \mathscr{I}_{Z} \rightarrow 0
$$

and the relations matrix is $\left(-f_{2}, f_{1}\right)$. It is easy to check that the complete intersection of $f_{1}$ and $f_{2}$ lies in generic position if and only if $\operatorname{deg} f_{i} \leq 2$. More generally:

Proposition 1.2. (a) $A$ subscheme $Z$ of length $s$ in $\mathbf{P}^{2}$ lies in generic position if and only if $0 \leq \alpha_{i j} \leq 2$ for a minimal resolution (1). (b) Moreover, in this situation, $r_{1} \leq r_{i} \leq r_{1}+1$ for all $1 \leq i \leq \nu$.

Proof. (a) Consider the long exact sequence of cohomology

$$
\begin{aligned}
0 & \rightarrow H^{0}\left(\mathscr{I}_{Z}(m)\right) \rightarrow H^{0}\left(\mathcal{O}_{\mathbf{P}^{2}}(m)\right) \rightarrow H^{0}\left(\mathcal{O}_{Z}(m)\right) \\
& \rightarrow H^{1}\left(\mathscr{I}_{Z}(m)\right) \rightarrow 0 .
\end{aligned}
$$

Denote $\operatorname{dim} H^{i}$ by $h^{i}$. Because $h^{0}\left(\mathcal{O}_{\mathbf{P}^{2}}(m)\right)=\left(\begin{array}{c}m+2 \\ 2\end{array}\right)$ and $h^{0}\left(\mathcal{O}_{Z}(m)\right)=s$, it is clear that $Z$ lies in generic position if and only if $\mathscr{I}_{Z}$ has "seminatural cohomology", i.e. for each $m \geq 0$, at most one of $H^{i}\left(\mathscr{I}_{Z}(m)\right), i=0,1,2$, is nonzero. Since $H^{0}\left(\mathscr{I}_{Z}(m)\right) \neq 0$ if and only if $m \geq r_{1}, \mathscr{I}_{Z}$ has seminatural cohomology if and only if $H^{1}\left(\mathscr{I}_{Z}(m)\right)=0$ for $m \geq r_{1}$. By (1) and the fact that $t_{\nu-1}>r_{\nu}$, this condition is equivalent to $t_{\nu-1} \leq r_{1}+2$.

(b) In this situation, since $t_{\nu-1}>r_{i}$ for all $i, r_{1} \leq r_{i}<t_{\nu-1} \leq r_{1}+2$ for all $i$.

REMARK 1.2.1. Fact (b) was proved in [7]. 
One of the advantages of generic position is that it is preserved under linkage. We say that two closed subschemes $Z, Z^{\prime}$ of $\mathbf{P}^{n}$, equidimensional and without embedded components, are linked via the complete intersection $X$ containing $Z$ and $Z^{\prime}$ if $I(Z)=I(X): I\left(Z^{\prime}\right)$ and $I\left(Z^{\prime}\right)=I(X)$ : $I(Z)$, where $I(Z)$ denotes the homogeneous graded ideal of $Z$, and so forth. The next proposition was essentially known to Apéry (see [5]) and has an elementary proof, which is provided in an appendix for lack of a reference.

Proposition 1.3. Let $Z$ be a projectively Cohen-Macaulay subscheme of $\mathbf{P}^{n}$ of codimension two, and let

$$
0 \rightarrow \sum_{i=1}^{\nu-1} \mathcal{O}_{\mathbf{P}^{n}}\left(-t_{i}\right) \stackrel{A}{\rightarrow} \sum_{j=1}^{\nu} \mathcal{O}_{\mathbf{P}^{n}}\left(-r_{j}\right) \stackrel{\left(f_{1}, \ldots, f_{\nu}\right)}{\rightarrow} \mathscr{I}_{Z} \rightarrow 0
$$

be a minimal projective resolution for the ideal sheaf, where $f_{i}$ is the ith maximal minor of $A$. Suppose $\left\{f_{i}, f_{j}\right\}$ forms a regular sequence of length two in $k\left[X_{0}, \ldots, X_{n}\right]$, and let $Z^{\prime}$ be the (projectively Cohen-Macaulay) subscheme of $\mathbf{P}^{n}$ linked to $Z$ via $\left\{f_{i}, f_{j}\right\}$. Then a relations matrix for a minimal resolution for $\mathscr{I}_{Z^{\prime}}$ is obtained by deleting columns $i$ and $j$ from $A$ and transposing.

REMARK 1.3.1. The liaison theorem ([9, Thm. 3.2]) follows directly from (1.3) and the fact that if $I$ is an ideal in $k\left[x_{0}, \ldots, x_{n}\right]$, a graded quotient of a polynomial ring, and $I$ contains a non-zero-divisor, then there exists a non-zero-divisor $x \in I$ belonging to a set of minimal generators for $I$.

REMARK 1.3.2. If we assume $i=\nu-1, j=\nu$ in (1.3), then $\alpha_{k l}^{\prime}=\alpha_{l k}$ for $1 \leq k \leq \nu-2,1 \leq l \leq \nu-1$.

COROllary 1.4. Let $Z$ be a set of points in $\mathbf{P}^{2}$ lying in generic position and $f_{1}, f_{2}$ two homogeneous forms with no common factor which belong to a minimal set of generators of the ideal $I(Z)=\sum_{m=0}^{\infty} H^{0}\left(\mathscr{I}_{Z}(m)\right)$. Let $Z^{\prime}$ be the scheme linked to $Z$ via $\left\{f_{1}, f_{2}\right\}$. Then $Z^{\prime}$ lies in generic position.

Proof. Follows from (1.2) and (1.3.2).

Next we find a lower bound on the number of generators $\nu$ of the graded ideal $I(Z)$, where $Z$ is a length $s$ subscheme of $\mathbf{P}^{2}$ lying in generic position. A set of generators must contain a basis for $H^{0}\left(\mathscr{I}_{Z}(r)\right)$, where $r$ 
is the least degree of a curve containing $Z$. Generic position implies that $r$ is the least integer such that $\left(\begin{array}{c}r+2 \\ 2\end{array}\right)>s$, and we have $\nu \geq k$ where $k=$ $h^{0}\left(\mathscr{I}_{Z}(r)\right)=\left(\begin{array}{c}r+2 \\ 2\end{array}\right)-s$. Using (1.2) we see that a minimal resolution for $\mathscr{I}_{Z}$ has the form

$$
\begin{aligned}
0 & \rightarrow \sum_{i=1}^{\nu-l-1} \mathcal{O}_{\mathbf{P}^{2}}(-r-1) \oplus \sum_{i=1}^{l} \mathcal{O}_{\mathbf{P}^{2}}(-r-2) \\
& \rightarrow \sum_{i=1}^{k} \mathcal{O}_{\mathbf{P}^{2}}(-r) \oplus \sum_{i=1}^{\nu-k} \mathcal{O}_{\mathbf{P}^{2}}(-r-1) \rightarrow \mathscr{I}_{Z} \rightarrow 0
\end{aligned}
$$

where $l=r+1-k$. Since $\nu-k$ and $\nu-l-1$ are nonnegative, $\nu \geq$ $\max \{k, r-k+2\}$. Set $N(s)=\max \{k, r-k+2\}$ (clearly $r$ and $k$ depend only on $s)$. Then we can compute

$$
\begin{aligned}
N(s) & =\max \{k, r-k+2\} \\
& =\max \left\{\left(\begin{array}{c}
r+2 \\
2
\end{array}\right)-s, r-\left(\begin{array}{c}
r+2 \\
2
\end{array}\right)+s+2\right\} \\
& =\left\{\begin{array}{cc}
r+1-s+\left(\begin{array}{c}
r+1 \\
2
\end{array}\right) & \text { if }\left(\begin{array}{c}
r+1 \\
2
\end{array}\right) \leq s \leq \frac{r(r+2)}{2} \\
r+2+s-\left(\begin{array}{c}
r+2 \\
2
\end{array}\right) & \text { if } \frac{r(r+2)}{2} \leq s<\left(\begin{array}{c}
r+2 \\
2
\end{array}\right) .
\end{array}\right.
\end{aligned}
$$

Therefore, $N(s)$, a number depending only on $s$, is a lower bound on the number of generators for the ideal $I(Z)$. To show this bound is sharp, we will exhibit for each integer $s$ a zero-dimensional scheme of length $s$ in generic position with exactly $N(s)$ ideal generators. By the following Lemma 1.5, having $N(s)$ ideal generators is an open condition on the set $Z$. It follows (1.7) that the general set of $s$ distinct points in $\mathbf{P}^{2}$ has $N(s)$ ideal generators.

LEMMA 1.5. Let $\mathscr{U}$ be the open dense subset of $\mathrm{Hilb}^{s}\left(\mathbf{P}^{2}\right)$ corresponding to schemes in generic position. For any integer $N$, the subset of $\mathscr{U}$ corresponding to schemes defined by at most $N$ equations is open in $\operatorname{Hilb}^{s}\left(\mathbf{P}^{2}\right)$.

Proof. The sheaf of differentials $\Omega_{\mathbf{P}^{2}}$ fits in the following exact sequence:

$$
0 \rightarrow \Omega_{\mathbf{P}^{2}} \rightarrow 3 \mathcal{O}_{\mathbf{P}^{2}}(-1) \stackrel{(x, y, z)}{\rightarrow} \mathcal{O}_{\mathbf{P}^{2}} \rightarrow 0
$$

where $\mathbf{P}^{2}=\operatorname{Proj} k[x, y, z]$. Let $Z \in \operatorname{Hilb}^{s}\left(\mathbf{P}^{2}\right)$, lying in generic position. Tensor the above sequence with $\mathscr{I}_{Z}(r+1)$, where $r$ is the least integer 
such that $\left(\begin{array}{c}r+2 \\ 2\end{array}\right)>s$. The long exact sequence of cohomology yields

$$
H^{0}\left(3 \mathscr{I}_{Z}(r)\right) \stackrel{(x, y, z)}{\rightarrow} H^{0}\left(\mathscr{I}_{Z}(r+1)\right) \rightarrow H^{1}\left(\Omega_{\mathbf{P}^{2}} \otimes \mathscr{I}_{Z}(r+1)\right) \rightarrow 0
$$

since $H^{1}\left(3 \mathscr{I}_{Z}(r)\right)=0$ by the seminatural cohomology of $\mathscr{I}_{Z}$. The dimension of $H^{1}\left(\Omega_{\mathrm{P}^{2}} \otimes \mathscr{I}_{Z}(r+1)\right)$ measures the number of generators of $I(Z)$ of degree $r+1$. Thus

$$
\nu(I(Z))=\left(\begin{array}{c}
r+2 \\
2
\end{array}\right)-s+h^{1}\left(\Omega_{\mathbf{P}^{2}} \otimes \mathscr{I}_{Z}(r+1)\right) .
$$

By the semicontinuity theorem ([8], Thm. III.12.8), $\nu(I(Z))$ is an upper semicontinuous function of $Z$ in $\mathscr{U}$.

LEMMA 1.6 (Buchsbaum, Eisenbud [2]). Let $R$ be a commutative noetherian ring, $F_{j}$ free $R$-modules. The complex

$$
0 \rightarrow F_{n} \stackrel{\varphi_{n}}{\rightarrow} F_{n-1} \stackrel{\varphi_{n-1}}{\rightarrow} F_{n-2} \rightarrow \cdots \rightarrow F_{0}
$$

is exact if and only if for $j=1,2, \ldots, n$,

(a) $\operatorname{rank} \varphi_{j}+\operatorname{rank} \varphi_{j-1}=\operatorname{rank} F_{j-1}$,

(b) the ideal of maximal minors of $\varphi_{j}$ contains a regular sequence of length $j$.

Proposition 1.7 (Geramita, Maroscia [6]). If $Z$ is a set of $s$ points in generic position in $\mathbf{P}^{2}$, then $\nu(I(Z)) \geq N(s)$. Moreover, equality holds for all $Z$ in an open dense subset of $\operatorname{Hilb}^{s}\left(\mathbf{P}^{2}\right)$.

Proof. The first statement is proved above. For the second, it remains to construct for each $s$ a length $s$ scheme $Z$ in generic position with $\nu(I(Z))=N(s)$.

Case 1. Suppose $\left(\begin{array}{c}r+1 \\ 2\end{array}\right) \leq s \leq r(r+2) / 2$ for some integer $r$. Set $m=r(r+2)-2 s, N=N(s)=r+1-s+\left(\begin{array}{c}r+1 \\ 2\end{array}\right)>0$. An easy calculation shows $0 \leq m \leq N-1$. Consider the $(N-1) \times N$ matrix

$$
A=\left(\begin{array}{ccc}
l_{11} & \cdots & l_{1 n} \\
\vdots & & \vdots \\
l_{m 1} & \cdots & l_{m N} \\
c_{m+1,1} & \cdots & c_{m+1, N} \\
\vdots & & \vdots \\
c_{N-1,1} & \cdots & c_{N-1, N}
\end{array}\right)
$$


where $\operatorname{deg} l_{i j}=1$ and $\operatorname{deg} c_{i j}=2$. If the entries of the matrix are chosen generally it is clear that at least two of the maximal minors $f_{1}, \ldots, f_{N}$ will share no common factor, and grade $\left\langle f_{1}, \ldots, f_{N}\right\rangle \geq 2$. Now (1.6) applies to show that the corresponding sequence (1) is exact with $\nu=N$, so that we have constructed a zero-dimensional scheme $Z$ with $A$ as relations matrix.

Counting degrees, we have $r_{1}=\cdots=r_{n}=2 N-m-2, t_{1}=\cdots=$ $t_{m}=2 N-m-1$, and $t_{m+1}=\cdots=t_{N-1}=2 N-m$. A calculation shows the length of $Z$ is $s$, and by (1.2) $Z$ lies in generic position. The resolution is minimal, so $\nu(I(Z))=N(s)$.

Case 2. Suppose $r(r+2) / 2 \leq s<\left(\begin{array}{c}r+2 \\ 2\end{array}\right)$ for some integer $r$. Set $m=\left(\begin{array}{c}r+2 \\ 2\end{array}\right)-s$ and $N=N(s)=s-\left(\begin{array}{c}r+2 \\ 2\end{array}\right)+r+2>0$. Note that $0 \leq m$ $\leq N$. Consider the $(N-1) \times N$ matrix

$$
A=\left(\begin{array}{cccccc}
c_{11} & \cdots & c_{1 m} & l_{1, m+1} & \cdots & l_{1 N} \\
\vdots & & \vdots & \vdots & & \vdots \\
c_{N-1,1} & \cdots & c_{N-1, m} & l_{N-1, m+1} & \cdots & l_{N-1, N}
\end{array}\right)
$$

where $\operatorname{deg} l_{i j}=1$ and $\operatorname{deg} c_{i j}=2$. As before, if the entries are chosen generally there exists a minimal projective resolution of an ideal sheaf $I_{Z}$ with $A$ as relations matrix. We have

$$
r_{1}=\cdots=r_{m}=N+m-2, r_{m+1}=\cdots=r_{N}=N+m-1,
$$

$t_{1}=\cdots=t_{N-1}=N+m$, showing that $Z$ consists of $s$ points in generic position, and $\nu(I(Z))=N(s)$.

Proposition 1.8. Let $Z$ be a set of $s$ points in generic position in $\mathbf{P}^{2}, r$ the least integer such that $\left(\begin{array}{c}r+2 \\ 2\end{array}\right)>s$. Then $\nu(I(Z)) \leq r+1$.

Proof. Let $Z$ be a length $s$ subscheme of $\mathbf{P}^{2}$, and $r$ the least degree of a curve containing $Z$. Let $A$ be a relations matrix for a minimal resolution (1) of $\mathscr{I}_{Z}$. We may arrange $A$ such that $\alpha_{11} \geq \alpha_{12} \geq \cdots \geq \alpha_{1 v}$; then $\operatorname{deg} f_{1}=r$ and $f_{1}$ is the determinant of a $(\nu-1) \times(\nu-1)$ matrix whose nonzero entries are of degree at least one. Therefore $r=\operatorname{deg} f_{1} \geq \nu(I(Z))$ -1 , i.e. $\nu(I(Z)) \leq r+1$. This is a theorem of Dubreil ([4], Thm. I).

For points in uniform position, the upper bound of (1.8) can be improved:

Proposition 1.9. Let $Z$ be a set of $s$ points in uniform position in $\mathbf{P}^{2}$, such that $\left(\begin{array}{c}r+1 \\ 2\end{array}\right)+1 \leq s \leq\left(\begin{array}{c}r+2 \\ 2\end{array}\right)-2$ for some $r$. Then $\nu(I(Z)) \leq r$. 
Proof. Let $k=\left(\begin{array}{c}r+2 \\ 2\end{array}\right)-s$; so that $2 \leq k \leq r$. Suppose $\nu(I(Z))=r+$ 1 , and let $A$ be the relations matrix of a minimal resolution (1) of $\mathscr{I}_{Z}$.

$$
A=\left(\begin{array}{ccc}
a_{11} & \cdots & a_{1, r+1} \\
\vdots & & \vdots \\
a_{r, 1} & \cdots & a_{r, r+1}
\end{array}\right)
$$

By (1.2), $0 \leq \alpha_{i j} \leq 2$, and $\alpha_{i j}=\operatorname{deg} a_{i j}$. We may assume $\alpha_{11} \geq \alpha_{12} \geq \cdots$ $\geq \alpha_{1, r+1}$; then we have

$$
\alpha_{i 1}=\alpha_{i 2}=\cdots=\alpha_{i k}=\alpha_{i, k+1}+1=\cdots=\alpha_{i, r+1}+1 .
$$

We may also assume $\alpha_{11} \leq \alpha_{21} \leq \cdots \leq \alpha_{r 1}$.

Since $\operatorname{deg} f_{r+1}=r+1$, we have

and thus

$$
\begin{aligned}
r+1 & =\alpha_{11}+\alpha_{22}+\cdots \alpha_{r r} \\
& =\alpha_{11}+\cdots+\alpha_{k l}+\left(\alpha_{k+1,1}-1\right)+\cdots+\left(\alpha_{r 1}-1\right) \\
& =\alpha_{11}+\cdots+\alpha_{r 1}-r+k
\end{aligned}
$$

$$
2 r-k+1=\alpha_{11}+\cdots+\alpha_{r 1} .
$$

By (2), $\alpha_{i 1} \geq 1$ for all $i$.

Suppose $\alpha_{k-1,1} \geq 2$. Then $2 \leq \alpha_{k-1,1} \leq \alpha_{k 1} \leq \cdots \leq \alpha_{r 1}$ and so $\alpha_{11}$ $+\cdots+\alpha_{r 1} \geq k-2+2(r-k+2)=2 r-k+2$, contradicting (3). Therefore, $1=\alpha_{k-1,1}=\alpha_{k-2,1}=\cdots=\alpha_{11}$.

By (2), $\alpha_{i j}=0$ for $1 \leq i \leq k-1, k+1 \leq j \leq r+1$. Since all entries of $A$ lie in the maximal homogeneous ideal $\left(X_{0}, X_{1}, X_{2}\right)$ of $k\left[X_{0}, X_{1}, X_{2}\right]$, $a_{i j}=0$ for $1 \leq i \leq k-1, k+1 \leq j \leq r+1$. $A$ has the following form:

$$
\left(\begin{array}{c|c|c|c}
* & A_{1} & 0 \\
\vdots & & k-1 \\
\hline \vdots & A_{2} & A_{3}
\end{array}\right\} \underbrace{r-k+1}_{k-1} \quad r-k+1
$$

Case 1. If $s=\left(\begin{array}{c}r+1 \\ 2\end{array}\right)+1$, then $k=r$, and the form of $A$ is

$$
\left(\begin{array}{c|c}
B & 0 \\
& \vdots \\
& 0 \\
a_{r, r+1}
\end{array}\right)
$$

where $\operatorname{deg} a_{r, r+1}=1$. The complete intersection of the curves det $B=0$ and $a_{r, r+1}=0$ lies in $Z$. Since $r \geq 2, \operatorname{deg}(\operatorname{det} B) \geq 3$, so $Z$ contains three 
collinear points (counted with multiplicity), a contradiction to the uniform position assumption.

Case 2. If $\left(\begin{array}{c}r+1 \\ 2\end{array}\right)+2 \leq s \leq\left(\begin{array}{c}r+2 \\ 2\end{array}\right)-2$, then $f_{1}=\left(\operatorname{det} A_{1}\right) \cdot\left(\operatorname{det} A_{3}\right)$, so $f_{1}$ is the composite of curves of degrees $k-1 \geq 1$ and $\nu-k \geq 1$. This contradicts the following fact:

LemMa 1.10 (Geramita, Maroscia [6], Thm. 3.4). Let $Z$ be a set of $s$ points in uniform position in $\mathbf{P}^{2}$ where $\left(\begin{array}{c}r+1 \\ 2\end{array}\right)+2 \leq s \leq\left(\begin{array}{c}r+2 \\ 2\end{array}\right)-1$. Then every curve of degree $r$ containing $Z$ is irreducible.

Proposition 1.11. Let $Z$ be a set of $s$ points in $\mathbf{P}^{2}$, and set

$$
N(s)=\left\{\begin{array}{ll}
r+1-s+\left(\begin{array}{c}
r+1 \\
2
\end{array}\right) & \text { if }\left(\begin{array}{c}
r+1 \\
2
\end{array}\right) \leq s \leq \frac{r(r+2)}{2} \\
r+2+s-\left(\begin{array}{c}
r+2 \\
2
\end{array}\right) & \text { if } \frac{r(r+2)}{2} \leq s<\left(\begin{array}{c}
r+2 \\
2
\end{array}\right)
\end{array} .\right.
$$

(a) If $Z$ lies in generic position, then $N(s) \leq \nu(I(Z)) \leq r+1$.

(b) If $Z$ lies in uniform position, then

$$
\begin{array}{ll}
N(s) \leq \nu(I(Z)) \leq r \quad \text { if }\left(\begin{array}{c}
r+1 \\
2
\end{array}\right)<s<\left(\begin{array}{c}
r+2 \\
2
\end{array}\right)-1 \\
N(s)=\nu(I(Z))=r+1 \quad \text { if } s=\left(\begin{array}{c}
r+1 \\
2
\end{array}\right) \text { or }\left(\begin{array}{c}
r+2 \\
2
\end{array}\right)-1 .
\end{array}
$$

Proof. Follows from (1.7), (1.8) and (1.9).

Corollary 1.12. (Geramita, Maroscia, Orecchia [6], [7]). (a) If Z lies in generic position and $s=\left(\begin{array}{c}r+1 \\ 2\end{array}\right)$ or $\left(\begin{array}{c}r+2 \\ 2\end{array}\right)-1$, then $\nu(I(Z))=r+1$. (b) If $Z$ lies in uniform position and $s=\left(\begin{array}{c}r+1 \\ 2\end{array}\right)+1$ or $\left(\begin{array}{c}r+2 \\ 2\end{array}\right)-2$, then $\nu(I(Z))=r$.

COROllary 1.13. If $Z$ lies in uniform position and $s=\left(\begin{array}{c}r+2 \\ 2\end{array}\right)-3$, then $r-1 \leq \nu(I(Z)) \leq r$.

REMARK 1.13.1. It follows from (1.11) that if $s \leq 11, s$ points in uniform position have exactly $N(s)$ ideal generators. In the next section we show that this statement fails for $s=12$.

2. A counterexample. The following result is related to the classical Cayley-Bacharach Theorem.

LemMa 2.1. Let $S, T$ be zero-dimensional subschemes of $\mathbf{P}^{2}$ linked by two curves $C, D$ of degrees $c$ and $d$, respectively, having no common components. Suppose $d \leq c+2$. Then $h^{0}\left(\mathscr{I}_{S}(d-3)\right)=h^{1}\left(\mathscr{I}_{T}(c)\right)$. 
Proof. Suppose $\mathscr{I}_{T}$ has minimal resolution

$$
0 \rightarrow \sum_{i=1}^{\nu-1} \mathcal{O}_{\mathbf{P}^{2}}\left(-t_{i}\right) \rightarrow \sum_{j=1}^{\nu} \mathcal{O}_{\mathbf{P}^{2}}\left(-r_{j}\right) \rightarrow \mathscr{I}_{T} \rightarrow 0 .
$$

Then linkage implies ([9], Prop. 2.5)

$$
\begin{aligned}
0 & \rightarrow \sum_{j=1}^{\nu} \mathcal{O}_{\mathbf{P}^{2}}\left(r_{j}-c-d\right) \\
& \rightarrow \sum_{i=1}^{\nu-1} \mathcal{O}_{\mathbf{P}^{2}}\left(t_{i}-c-d\right) \oplus \mathcal{O}_{\mathbf{P}^{2}}(-c) \oplus \mathcal{O}_{\mathbf{P}^{2}}(-d) \rightarrow \mathscr{I}_{S} \rightarrow 0
\end{aligned}
$$

is a projective resolution for $\mathscr{I}_{S}$. By the long exact sequences of cohomology,

$$
\begin{aligned}
h^{0}\left(\mathscr{I}_{S}(d-3)\right) & =\sum_{i=1}^{\nu-1} h^{0}\left(\mathcal{O}_{\mathbf{P}^{2}}\left(t_{i}-c-3\right)\right)-\sum_{j=1}^{\nu} h^{0}\left(\mathcal{O}_{\mathbf{P}^{2}}\left(r_{j}-c-3\right)\right) \\
& =\sum_{i=1}^{\nu-1} h^{2}\left(\mathcal{O}_{\mathbf{P}^{2}}\left(c-t_{i}\right)\right)-\sum_{j=1}^{\nu} h^{2}\left(\mathcal{O}_{\mathbf{P}^{2}}\left(c-r_{j}\right)\right) \\
& =h^{1}\left(\mathscr{I}_{T}(c)\right) .
\end{aligned}
$$

LeMma 2.2. Suppose $P_{1}, \ldots, P_{13}$ are points in $\mathbf{P}^{2}$ such that no three are collinear, and suppose $\left\{Q_{1}, Q_{2}, Q_{3}\right\}$ is linked to $\left\{P_{1}, \ldots, P_{13}\right\}$ via two quartic curves. Then every subset of $\left\{Q_{1}, Q_{2}, Q_{3}, P_{1}, \ldots, P_{10}\right\}$ imposes independent conditions on quartics.

Proof. Set $T=\left\{Q_{1}, Q_{2}, Q_{3}, P_{1}, \ldots, P_{10}\right\}$ and $S=\left\{P_{11}, P_{12}, P_{13}\right\}$ in (2.1). Since $P_{11}, P_{12}, P_{13}$ are not collinear, $h^{1}\left(\mathscr{I}_{T}(4)\right)=0$, i.e. $T$ imposes independent conditions on quartics. Since each point imposes at most one condition, it follows that every subset of $T$ imposes independent conditions on quartics.

According to (1.13) a set of 12 points in uniform position must have either 3 or 4 ideal generators. We know almost all have 3 ideal generators (1.7). If $\nu(I(Z))=3$, then the minimal resolution for $\mathscr{I}_{Z}$ must be

$$
0 \rightarrow 2 \mathcal{O}_{\mathbf{p}^{2}}(-6) \stackrel{A}{\rightarrow} 3 \mathcal{O}_{\mathbf{P}^{2}}(-4) \stackrel{\left(f_{1} f_{2} f_{3}\right)}{\rightarrow} \mathscr{I}_{Z} \rightarrow 0
$$

and $A$ has form

$$
\left(\begin{array}{lll}
c_{11} & c_{12} & c_{13} \\
c_{21} & c_{22} & c_{23}
\end{array}\right)
$$

where $\operatorname{deg} c_{i j}=2$. 
Suppose there are two quartic curves with no common component containing $Z$. We may assume they are given by $f_{1}=0$ and $f_{2}=0$. By (1.3), $Z$ is linked via $f_{1}$ and $f_{2}$ to the complete intersection of the two conics $c_{13}=0$ and $c_{23}=0$.

EXAMPle 2.3. A set of 12 points in uniform position in $\mathbf{P}^{2}$ with $\nu(I(Z))=4$.

We will construct a set $Z$ in uniform position that is linked via two quartics to four points, three of which are collinear. By the preceding argument, we conclude $\nu(I(Z))=4$.

The following criterion for uniform position will be used below.

Lemma 2.4 (Brun, [1]). A set of points $Z$ in $\mathbf{P}^{2}$ is in uniform position if and only if

(a) at most $\left(\begin{array}{c}r+2 \\ 2\end{array}\right)-1$ points of $Z$ lie on a degree $r$ curve, for each $r$, and

(b) for each $z \in Z$, there exists a degree d curve $Y$ such that $Y \cap Z=$ $Z \backslash\{z\}$, where $d$ is the smallest integer such that $\left(\begin{array}{c}d+2 \\ 2\end{array}\right) \geq s$.

Construction. Fix a nonsingular quartic $C$ in $\mathbf{P}^{2}$ and three distinct collinear points $Q_{1}, Q_{2}, Q_{3}$ on $C$. We will show that the general quartic passing through $Q_{1}, Q_{2}, Q_{3}$, intersects $C$ in $Q_{1}, Q_{2}, Q_{3}, P_{1}, \ldots, P_{13}$ such that any twelve of $P_{1}, \ldots, P_{13}$ lie in uniform position.

The family of quartics in $\mathbf{P}^{2}$ is parametrized by $\mathbf{P}^{14}$, and an 11-dimensional subfamily $S$ passes through $Q_{1}, Q_{2}, Q_{3}$, since it is clear that none of these three points is a base point for quartics through the other two.

The quartics in $S$ missing the point $Q_{4}$, the residual intersection of $C$ and the line $L$ connecting $Q_{1}, Q_{2}, Q_{3}$, form again an 11-dimensional family $S^{\prime}$. Suppose $C^{\prime} \in S^{\prime}$ and $C \cap C^{\prime}=\left\{Q_{1}, Q_{2}, Q_{3}, P_{1}, \ldots, P_{13}\right\}$, counting multiplicities, and that $P_{1}, P_{2}, P_{3}$ are collinear. None of the $P_{i}$ lie on $L . P_{3}$ is not a base point for quartics through $\left\{Q_{1}, Q_{2}, Q_{3}, P_{1}, P_{2}\right\}$ : for example, set $L_{1}=\overline{P_{1} Q_{i}}$, where we choose $Q_{i} \notin \overline{P_{1} P_{2}}$, and $L_{2}, L_{3}, L_{4}$ lines containing $P_{2}, Q_{j}, Q_{k}$, respectively, where $\{i, j, k\}=\{1,2,3\}$. Then there exists a quartic $L_{1} L_{2} L_{3} L_{4}$ containing $\left\{Q_{1}, Q_{2}, Q_{3}, P_{1}, P_{2}\right\}$ but not $P_{3}$. Similar arguments show that none of the six points $\left\{Q_{1}, Q_{2}, Q_{3}\right.$, $\left.P_{1}, P_{2}, P_{3}\right\}$ is a base point for quartics through the other five, so that the six points induce independent conditions on quartics.

Thus there are 8 dimensions of quartics through $\left\{P_{1}, P_{2}, P_{3}\right.$, $\left.Q_{1}, Q_{2}, Q_{3}\right\}$. The choice of a set of collinear points $\left\{P_{1}, P_{2}, P_{3}\right\}$ on $C \backslash L$ is 2-dimensional (since there is a finite choice (four) of three-point sets 
associated with each line in $\mathbf{P}^{2}$ ). Therefore there is a 10-dimensional set of quartics $C^{\prime}$ belonging to $S^{\prime}$ such that the remaining 13 points of the complete intersection $C \cap C^{\prime}$ contain three collinear points. We conclude that the general quartic through $Q_{1}, Q_{2}, Q_{3}$ is such that the residual intersection with $C$ does not contain three collinear points.

Showing the general residual intersection contains no six on a conic and no ten on a cubic requires similar arguments; we argue only the latter. Suppose $C^{\prime}$ is a general quartic such that $C \cap C^{\prime}=\left\{Q_{1}, Q_{2}, Q_{3}\right.$, $\left.P_{1}, \ldots, P_{13}\right\}$ where $\left\{P_{1}, \ldots, P_{10}\right\}$ are situated on a cubic. Since no three of $P_{1}, \ldots, P_{13}$ are collinear, $(2.2)$ applies to show $\left\{Q_{1}, \ldots, P_{10}\right\}$ imposes independent conditions on quartics, so that the family of quartics through $\left\{Q_{1}, \ldots, P_{10}\right\}$ is one-dimensional. The family of 10 -point sets on $C$ lying on a cubic is at most 9-dimensional, therefore the family of quartics through $Q_{1}, Q_{2}, Q_{3}$ such that the residual 13 points of $C \cap C^{\prime}$ contain 10 cocubic points is at most 10 -dimensional. We conclude that the general quartic through $Q_{1}, Q_{2}, Q_{3}$ intersects $C$ in 13 residual points that have no three collinear, no six on a conic, and no ten on a cubic.

Let $C^{\prime}$ be such a general quartic. We will show that any twelve of $P_{1}, \ldots, P_{13}$ satisfy the criterion of (2.4). Condition (a) is verified; for (b) we reason as follows. Given any point $z$ among a subset of twelve, choose a cubic containing nine of the rest and a line containing the other two. The point $z$ cannot lie on this reducible quartic $Y$, therefore $Y \cap Z=Z \backslash\{z\}$. Such a set $Z$ of twelve points lies in uniform position and is linked via $C$ and $C^{\prime}$ to a set of four points, three of which are collinear, so $\nu(I(Z))=4$.

REMARK 2.4.1. It is clear, at least if char $k=0$, that by Bertini's theorem the general such $C^{\prime}$ intersects $C$ transversally, i.e. in the terminology of [6], provides sets of 12 points in "transversal uniform position" and $\nu=4$.

Appendix. We present a proof of (1.3). Let $R$ be a commutative ring and

$$
A=\left(\begin{array}{ccc}
a_{11} & \cdots & a_{1 \nu} \\
\vdots & & \vdots \\
a_{\nu-1,1} & \cdots & a_{\nu-1, \nu}
\end{array}\right)
$$

be a matrix whose entries lie in $R$. Set

$$
f_{i}=(-1)^{i+1} \operatorname{det}\left(\begin{array}{c}
(\nu-1) \times(\nu-1) \text { matrix } \\
\text { obtained by deleting } \\
\text { column } i
\end{array}\right)
$$




$$
\Gamma_{i j}^{k}=(-1)^{i+j+k+1} \operatorname{det}\left(\begin{array}{c}
(\nu-2) \times(\nu-2) \text { matrix } \\
\text { obtained by deleting } \\
\text { columns } i, j \text { row } k
\end{array}\right)
$$

LEMMA 1. If $i<j$, then

$$
\left(\Gamma_{i j}^{1}, \ldots, \Gamma_{i j}^{\nu-1}\right) \cdot A=(\underset{i}{0, \ldots, 0, \underset{j}{f}, 0, \ldots, 0, \underset{j}{f}, 0, \ldots, 0)})
$$

LEMMA 2. If $1 \leq i<j \leq \nu, l \neq i$ or $j$, then

$$
\Gamma_{i j}^{k} f_{l}=\Gamma_{i l}^{k} f_{j}-\Gamma_{j l}^{k} f_{i} \text { for } k=1, \ldots, \nu-1 .
$$

Proof. For $l \neq i$ or $j$, multiply the equation in Lemma 1 on the right by the matrix

$$
B_{l}=\left(\begin{array}{cccc}
\Gamma_{1 l}^{1} & \Gamma_{1 l}^{2} & \cdots & \Gamma_{l l}^{\nu-1} \\
\vdots & \vdots & & \vdots \\
\Gamma_{l-1, l}^{1} & \Gamma_{l-1, l}^{2} & \cdots & \Gamma_{l-1, l}^{\nu-1} \\
0 & 0 & \cdots & 0 \\
-\Gamma_{l+1, l}^{1} & -\Gamma_{l+1, l}^{2} & \cdots & -\Gamma_{l+1, l}^{\nu-1} \\
\vdots & \vdots & & \vdots \\
-\Gamma_{\nu l}^{1} & -\Gamma_{\nu l}^{2} & \cdots & -\Gamma_{\nu l}^{\nu-1}
\end{array}\right)
$$

LEMMA 3. Let $(R, \mathfrak{M})$ be a regular local ring, $A a(\nu-1) \times \nu$ matrix with entries in $\mathfrak{M}$. If $\left\{f_{i}, f_{j}\right\}$ is a regular sequence in $\mathfrak{M}$, then $\left(f_{i}, f_{j}\right)$ : $\left(f_{1}, f_{2}, \ldots, f_{\nu}\right)=\left(\Gamma_{i j}^{1}, \ldots, \Gamma_{i j}^{\nu-1}\right)$.

Proof. By Lemma 2, $\left(\Gamma_{i j}^{1}, \ldots, \Gamma_{i j}^{\nu-1}\right) \subseteq\left(f_{i}, f_{j}\right):\left(f_{1}, \ldots, f_{\nu}\right)$. On the other hand, let $r \in\left(f_{i}, f_{j}\right):\left(f_{1}, \ldots, f_{\nu}\right)$. Then

$$
\left(\begin{array}{c}
f_{1} \\
\vdots \\
f_{\nu}
\end{array}\right) r=C\left(\begin{array}{c}
f_{i} \\
f_{j}
\end{array}\right)
$$

where

$$
C=\left(\begin{array}{cc}
c_{11} & c_{12} \\
\vdots & \vdots \\
c_{\nu 1} & c_{\nu 2}
\end{array}\right), \quad c_{i j} \in R
$$


and we may assume $c_{i 1}=r, c_{i 2}=0, c_{j 1}=0, c_{j 2}=r$. Multiply on the left by $A$ :

$$
0=A C\left(\begin{array}{l}
f_{i} \\
f_{j}
\end{array}\right) .
$$

$A C$ is a $(\nu-1) \times 2$ matrix whose first column is divisible by $f_{j}$ and whose second column is divisible by $f_{i}$, since $\left\{f_{i}, f_{j}\right\}$ form a regular sequence. So write

$$
A C=\left(\begin{array}{cc}
d_{1} f_{j} & -d_{1} f_{i} \\
\vdots & \vdots \\
d_{\nu-1} f_{j} & -d_{\nu-1} f_{i}
\end{array}\right)
$$

where $d_{k} \in R$ for all $k=1, \ldots, \nu-1$. Multiplying on the left by the matrix $\left(\Gamma_{i j}^{1}, \ldots, \Gamma_{i j}^{\nu-1}\right)$ and using Lemma 1 , we get

$$
r=\sum_{k=1}^{\nu-1} d_{k} \Gamma_{i j}^{k} \in\left(\Gamma_{i j}^{1}, \ldots, \Gamma_{i j}^{\nu-1}\right)
$$

Proposition (1.3) follows from

LEMMA 4. Let $(R, \mathscr{M})$ be a regular local ring, I an ideal with a minimal resolution

$$
0 \rightarrow R^{\nu-1} \stackrel{A}{\rightarrow} R^{\nu} \stackrel{\left(\begin{array}{c}
f_{1} \\
\vdots \\
f_{\nu}
\end{array}\right)}{\rightarrow} I \rightarrow 0,
$$

$f_{i}$ the maximal minors of $A$. Suppose $\left\{f_{i}, f_{j}\right\}$ is a regular sequence in $R$, and let $J=\left(f_{i}, f_{j}\right):\left(f_{1}, \ldots, f_{\nu}\right)$ be the ideal linked to $I$ by $\left\{f_{i}, f_{j}\right\}$. Then $J$ has $a$ minimal resolution

$$
0 \rightarrow R^{\nu-2} \stackrel{B}{\rightarrow} R^{\nu-1} \stackrel{\left(\begin{array}{c}
\Gamma_{t \jmath}^{\nu-1} \\
\vdots \\
\Gamma_{t J}^{1}
\end{array}\right)}{\rightarrow} J \rightarrow 0
$$

where $B$ is the transpose of the matrix obtained by eliminating columns $i$ and $j$ from $A$.

Proof. Easy to check that

$$
B\left(\begin{array}{c}
\Gamma_{i j}^{\nu-1} \\
\vdots \\
\Gamma_{i j}^{1}
\end{array}\right)=0
$$


Therefore

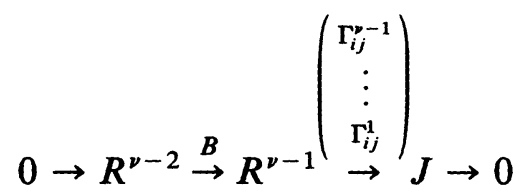

is a complex. Rank $A=\nu-1 \Rightarrow \operatorname{rank} B=\nu-2$, so the complex is exact on the left. It is exact on the right by Lemma 3. The grade of $J$ is 2 by ([9], Prop. 1.3), so (1.6) applies to prove exactness in the middle. Since the entries of $B$ lie in $\mathscr{M}$, this exact sequence is a minimal resolution of $J$.

Note added in proof. An example with the properties of Example 2.3, constructed by different techniques, is included in a later version of [6].

\section{REFERENCES}

[1] J. Brun, Les fibrés de rang deux sur $\mathbf{P}_{2}$ et leurs sections, Bull. Soc. Math. France, 108 (1979), 457-473.

[2] D. Buchsbaum and D. Eisenbud, What makes a complex exact? J. Algebra, 25 (1973), 259-268.

[3] L. Burch, A note on ideals of homological dimension one in local domains, Proc. Camb. Phil. Soc., 63, (1967), 661-662.

[4] P. Dubreil, Sur quelques propriétiés des systemes de points dans le plan et des courbes gauches algebriques. Bull. Soc. Math. France, 61 (1933), 258-283.

[5] F. Gaeta, Quelques progrès recents dans la classification des variétiés algébriques d'un espace projectif. Deuxieme Colloque de Geometrie Algébrique Liège, C.B.R.M. (1952), 145-181.

[6] A. V. Geramita and P. Maroscia, The ideal of forms vanishing at a finite set of points in $\mathbf{P}^{n}$, Queen's Mathematical Preprint No. 1981-5.

[7] A. V. Geramita and F. Orecchia, Minimally generating ideals defining certain tangent cones, J. Algebra, 78 (1982), 36-57.

[8] R. Hartshorne, Algebraic Geometry, Graduate Texts in Math. 52, Berlin, Heidelberg, New York: Springer, 1977.

[9] C. Peskine and L. Szpiro, Liaison des variétiés algébriques I, Inventiones Math., 26 (1974), 271-302.

Received April 6, 1984.

Michigan STate University

EAST LANSING, MI 48824 



\section{PACIFIC JOURNAL OF MATHEMATICS EDITORS}

V. S. VARAdarajan (Managing Editor)
University of California
Los Angeles, CA 90024
CHARLES R. DePrima
California Institute of Technology
Pasadena, CA 91125
R. FINN
Stanford University
Stanford, CA 94305

V. S. VARADARAJAN (Managing Editor)

Hermann FlaschKa

University of Arizona

Tucson, AZ 85721

RAMESH A. GANGOlli

University of Washington

Seattle, WA 98195

ROBION KIRBY

University of California

Berkeley, CA 94720
C. C. MOORE

University of California

Berkeley, CA 94720

H. SAMELSON

Stanford University

Stanford, CA 94305

Harold Stark

University of California, San Diego

La Jolla, CA 92093

\section{ASSOCIATE EDITORS}
R. ARENS
E. F. BECKENBACH
B. H. NeumanN
F. WOLF
K. Yoshida (1906-1982)

\section{SUPPORTING INSTITUTIONS}

\author{
UNIVERSITY OF ARIZONA \\ UNIVERSITY OF BRITISH COLUMBIA \\ CALIFORNIA INSTITUTE OF TECHNOLOGY \\ UNIVERSITY OF CALIFORNIA \\ MONTANA STATE UNIVERSITY \\ UNIVERSITY OF NEVADA, RENO \\ NEW MEXICO STATE UNIVERSITY \\ OREGON STATE UNIVERSITY
}

UNIVERSITY OF OREGON UNIVERSITY OF SOUTHERN CALIFORNIA

STANFORD UNIVERSITY

UNIVERSITY OF HAWAII

UNIVERSITY OF TOKYO

UNIVERSITY OF UTAH

WASHINGTON STATE UNIVERSITY

UNIVERSITY OF WASHINGTON 


\section{Pacific Journal of Mathematics}

\section{Vol. 120, No. $1 \quad$ September, 1985}

Ulrich F. Albrecht, A note on locally $A$-projective groups $\ldots \ldots \ldots \ldots \ldots 1$

Marilyn Breen, A Krasnosel'skiü-type theorem for unions of two starshaped

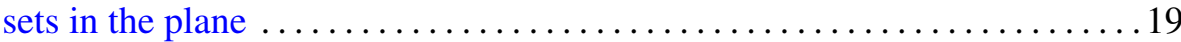

Anthony Carbery, Sun-Yung Alice Chang and John Brady Garnett,

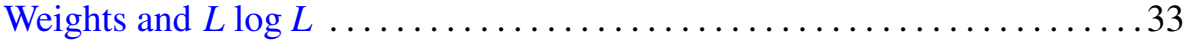

Joanne Marie Dombrowski, Tridiagonal matrix representations of cyclic

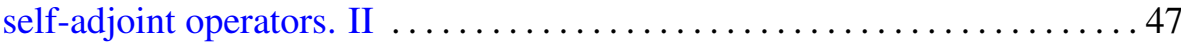

Heinz W. Engl and Werner Römisch, Approximate solutions of nonlinear random operator equations: convergence in distribution $\ldots \ldots \ldots \ldots \ldots 55$

P. Ghez, R. Lima and J. E. Roberts, $W^{*}$-categories $\ldots \ldots \ldots \ldots \ldots \ldots$

Barry E. Johnson, Continuity of homomorphisms of Banach $G$-modules . . 111 Elyahu Katz and Sidney Allen Morris, Free products of topological groups

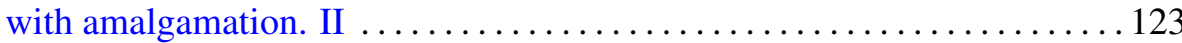

Neal I. Koblitz, $p$-adic integral transforms on compact subgroups of $\mathbf{C}_{p} \ldots 131$ Albert Edward Livingston, A coefficient inequality for functions of positive real part with an application to multivalent functions $\ldots \ldots \ldots 139$

Scott Carroll Metcalf, Finding a boundary for a Hilbert cube manifold

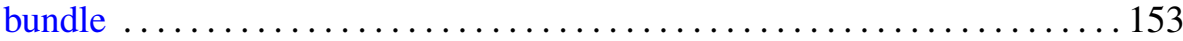

Jack Ray Porter and R. Grant Woods, When all semiregular $H$-closed extensions are compact ........................

Francisco José Ruiz and José Luis Torrea, A unified approach to Carleson measures and $A_{p}$ weights. II $\ldots \ldots \ldots \ldots \ldots \ldots \ldots \ldots \ldots \ldots \ldots \ldots \ldots$

Timothy DuWayne Sauer, The number of equations defining points in general position

John Brendan Sullivan, Universal observability and codimension one subgroups of Borel subgroups

Akihito Uchiyama, Extension of the Hardy-Littlewood-Fefferman-Stein inequality 